\title{
El impacto de la crisis de la construcción en las relaciones intergrupales dentro del sector
}

Impact of the construction crisis in the intergroup relations within the sector

\author{
Beatriz González-Martín \\ Doctoranda. Universidad de Almería. España. \\ bgm620@ual.es
}

\section{Pablo Pumares Fernández}

Profesor Titular. Universidad de Almería.

ppumares@ual.es

\section{Antonio J. Rojas Tejada}

Profesor Titular. Universidad de Almería.

arojas@ual.es

\section{RESUMEN}

El sector de la construcción ha sido uno de los motores del periodo de bonanza económica vivido en España durante la última década. La expansión de este sector facilitó la contratación de numerosos trabajadores autóctonos e inmigrantes. Actualmente, la crisis ha provocado una alarmante destrucción de empleos en dicho sector. Esto está ocasionando importantes repercusiones socioeconómicas y psicosociales en los trabajadores. El presente trabajo analiza si las dificultades económicas a las que se enfrentan los trabajadores de la construcción y la creciente competencia por los escasos puestos de trabajo están influyendo en las relaciones intergrupales en el sector. A partir de los discursos obtenidos de los principales implicados, mediante la realización de entrevistas en profundidad y grupos de discusión en la provincia de Almería, se evalúan las relaciones entre los grupos de trabajadores, en términos de hostilidad y conflicto hacia los trabajadores inmigrantes.

\section{ABSTRACT}

The construction sector has been one of the main driving forces of the prosperous economic period during the last decade in Spain. The expansion of this sector enabled the hiring of many native Spanish as well as immigrant workers. Lately, however, the economic crisis has caused alarming job destruction in this sector. This has resulted in a great socioeconomic and psychosocial impact on workers. The present paper analyses whether the economic difficulties that the construction workers are facing, and the increasing competition for the scarce jobs created at that time, are influencing the social relations between the groups belonging to the sector. The intergroup relations between workers are evaluated in terms of hostility and conflict with regard to immigrant workers through the statements gathered from the main actors involved, using in-depth interviews and discussion groups in Almería.

PALABRAS CLAVE

inmigración | mercado laboral | competencia | amenaza | relaciones sociales

KEYWORDS

immigration | labour market | competition | threat | social relationships

\section{Introducción}

\subsection{El sector de la construcción}

Desde el año 1996 hasta el 2007, España ha vivido una de las mayores épocas de bonanza económica de su historia reciente. Tras estos años de esplendor, de manera repentina, se vio sumida en una profunda crisis económica, en la que todavía se encuentra. 
El desarrollo económico de estos años estuvo conectado con el intenso incremento que se produjo en la creación de puestos de trabajo, en el que el sector de la construcción fue uno de los principales responsables. España alcanzó su máximo histórico de trabajadores ocupados con más de 20,5 millones en el tercer trimestre de del año 2007 (Encuesta de Población Activa). La construcción fue el sector que más incrementó en términos relativos el número de trabajadores en alta laboral en los años de expansión económica, alcanzando los 2.542.900 en el año 2007 (Boletín de Estadísticas Laborales), lo que supuso un crecimiento del 52,2\% entre el año 2000 y el 2007. En Andalucía, este aumento fue aún superior, alcanzando el 72,3\% para el mismo período (Anuario de Estadísticas Laborales). En la provincia de Almería, siguiendo esta misma tendencia, se observa que ese mismo año el 15\% de los trabajadores afiliados a la Seguridad Social pertenecían al gremio de la construcción.

El sector de la construcción durante la última década se convirtió en el "sector de la oportunidad laboral". La necesidad de cubrir los puestos de trabajo que se generaban aumentó a tal ritmo que la mano de obra autóctona comenzó a ser insuficiente. Los trabajadores inmigrantes empezaron a ocupar el excedente de puestos de trabajo que estaban surgiendo. Este aumento en la demanda de trabajadores dentro del sector repercutió muy positivamente en las condiciones laborales que se ofrecían a los trabajadores, particularmente los salarios. La Encuesta Anual de Costes Labores (Instituto Nacional de Estadística), por ejemplo, muestra el superior incremento experimentado por los salarios en el sector de la construcción respecto a la industria y al sector servicios entre los años 2001 y 2007.

Sin embargo, a partir de finales de 2007 la situación económica española empieza a desequilibrarse. El sector de la construcción ya desde mediados de 2006 había empezado a mostrar claros síntomas de agotamiento. Esto se unió al colapso de las hipotecas subprime en Estados Unidos durante el verano de 2007, que arrastró a buena parte del sistema bancario internacional, desencadenando una profunda crisis mundial. En España, esto precipitaría el estallido de la anunciada burbuja inmobiliaria, lo que agravaría las repercusiones sobre la economía nacional, en particular sobre el sector de la construcción (Clemente y García 2009).

Los datos de la EPA reflejan claramente el gran impacto que la crisis en el sector de la construcción ha tenido sobre el empleo. El número de parados a nivel nacional en el sector se multiplicó por cuatro, pasando de 174.600 parados en el primer trimestre de 2007 a 743.700 en 2009, lo que significó que en solo dos años se perdió todo el crecimiento ganado desde el año 2000 (González-Martín, Pumares y Rojas 2010).

El comportamiento del empleo, tanto en Andalucía como en la provincia de Almería, siguió esa misma línea. Entre 2007 y 2010 el número de ocupados en Andalucía descendió más de un 11\%. Almería, por su parte, en ese mismo periodo, acusó una caída del 15\% (EPA 2011).

Desde el año 2000, Almería ha sido la provincia andaluza que en términos relativos ha recibido mayor número de inmigrantes, registrando la tasa de inmigración más elevada, que alcanzó el 4,40\% en el año 2004 (Estadísticas de Variaciones Residenciales (Padrón Municipal). Por otro lado, con la llegada de la crisis destaca como la provincia andaluza que desde 2007 hasta 2010 ha perdido un mayor número de trabajadores ocupados en el sector de la construcción (75\%) en relación con el resto de las provincias andaluzas que mostraron descensos más moderados (EPA 2011). Estos datos señalaron a esta provincia como el lugar más idóneo para el desarrollo del trabajo realizado en el presente estudio.

Este enorme impacto de la crisis en la pérdida de empleo tanto de nacionales como de extranjeros, ha supuesto un grave deterioro de las condiciones laborales en el sector. Sin embargo, al comparar la tasa de paro referente al ámbito nacional presentada por la población inmigrante y por la autóctona en dicho sector en 2009, observamos que la de los trabajadores extranjeros se había incrementado casi cinco veces respecto al año 2007, pasando del 8\% al 37\% (EPA 2009). Igualmente, los datos reflejaban un aumento en la tasa de paro de los trabajadores españoles muy elevado, sin embargo, aunque se había multiplicado casi por cuatro en estos dos años (del 5,7\% en 2007 al 22,4\% en 2009), no alcanzó 
parámetros tan negativos como la de la población inmigrante (González-Martín, Pumares y Rojas 2010).

\subsection{El nuevo contexto económico y social}

En la actualidad y como consecuencia de la magnitud de la crisis que está sufriendo el sector de la construcción en España, con condiciones laborales más precarias y menores oportunidades de empleo para todos, cabe esperar que existan repercusiones negativas no solo en el ámbito económico sino también en las relaciones de convivencia que mantienen los grupos que forman el sector.

Portes y Boröcz (1992) señalan la importancia que el contexto cobra en las relaciones y las oportunidades laborales de los trabajadores inmigrantes, distinguiendo también según la cualificación y la clase social. Así, respecto a los trabajadores de menor cualificación, estos autores destacan sus dificultades de integración en el mercado laboral del país receptor cuando se tenían que desenvolver en un ambiente de acogida hostil. Mientras que estos trabajadores en el contexto hostil se concentran en el escalón inferior del mercado laboral (sector secundario), los mismos trabajadores al encontrarse en ambientes más neutros o favorables llegarían incluso a realizar actividades empresariales que les permitirían alcanzar posiciones de liderazgo social y político dentro de su grupo. El cambio de ciclo económico puede entenderse, en este sentido, como una modificación sustancial del contexto de acogida hacia un ambiente más hostil, lo que debería tener repercusiones negativas sobre las condiciones sociales y laborales de los trabajadores inmigrantes del sector. Diversos estudios, tanto internacionales como nacionales, que han puesto de manifiesto efectos de la nueva situación económica sobre las actitudes y las opiniones de la población autóctona hacia la población inmigrante, apoyan esta idea.

Desde una perspectiva muy global, el informe denominado Migration and Global Recession realizado en 2009 por la BBC Word Service y the Migration Policy Institute de Washington analiza los efectos que la recesión económica está causando en la población inmigrante de diferentes países entre los que se encuentra España. Dicho estudio apunta que uno de los efectos de la recesión económica es la creciente hostilidad que está apareciendo hacia esta población. El informe define esta hostilidad como una forma más de vulnerabilidad de la población inmigrante (Fix y otros 2009).

Como muestra de la preocupación en el ámbito europeo, el proyecto Living together (Cea y Valles 2010), pretende promover un discurso de tolerancia y consolidar una ciudadanía europea alejada del racismo y la xenofobia, y presenta una comparativa sobre la convivencia en seis países de la Unión Europea: Finlandia, Holanda, Irlanda, Portugal, España y Suecia. De este proyecto destacan dos resultados: por un lado, el cambio de actitud demostrado por la población más joven a raíz de la situación de crisis económica, cuando esta población, hasta el momento, había mostrado las opiniones más favorables hacia la inmigración, y, por otro lado, el despunte de España como el país que hace mayor hincapié en las repercusiones negativas de la inmigración en el mercado laboral.

Centrándonos en España, encontramos estudios como la Evolución del racismo y la xenofobia en España. Se trata de una investigación longitudinal iniciada en 2005 y realizada anualmente hasta nuestros días por el Observatorio Español del Racismo y la Xenofobia (OBERAXE). Recoge los principales indicadores relacionados con las opiniones de los españoles respecto al racismo y a la xenofobia; debate que se plantea en torno al fenómeno de la inmigración. Uno de los indicadores más significativos es el de la actitud de los españoles ante los efectos de la inmigración en el mercado laboral. Este indicador ha cobrado especial relevancia a partir del año 2008 con la llegada de la crisis. En el estudio se evalúa la propensión que existe por parte de los nacionales a culpar a los inmigrados de algunos fenómenos sociales como el desempleo, la bajada de los salarios, la pérdida de calidad en los servicios sociales, etc. (Cea y Valles 2009).

Dentro del ámbito laboral específicamente, González Enríquez (2008) analiza las actitudes de los 
trabajadores autóctonos de acuerdo al sector de ocupación al que pertenecen. Este trabajo, realizado en la última etapa del boom económico español, desvela la creciente preocupación de los trabajadores autóctonos de la construcción por la decadencia del sector y prevén la aparición de competencia por los puestos de trabajo. Además muestran cierta negatividad hacia los trabajadores inmigrantes y no descartan el brote de conflictos causados por la aceptación de éstos de rebajas de los salarios y peores condiciones laborales.

También se reflejan los cambios de actitud derivados de la crisis en el estudio Opiniones y actitudes de los andaluces ante la inmigración (OPIA) realizado entre 2005 y 2011 en la Comunidad Andaluza. En su última entrega (Rinken, Escobar y Velasco 2011) se resalta la importancia que la economía y la crisis toman en la percepción del fenómeno migratorio y la pérdida de vigencia de los demás debates a la hora de valorar dicho fenómeno. Del mismo modo, se advierte una mayor tendencia hacia discursos que presentan percepciones de agravio comparativo entre los trabajadores españoles y los extranjeros y el crecimiento de manifestaciones de hostilidad hacia ellos.

Dado el mayor impacto sufrido por el sector de la construcción y la notable presencia en el mismo de trabajadores inmigrantes, debemos esperar, siguiendo a Portes y Böröcz (1992), una repercusión negativa sobre la posición de los trabajadores en general, que impulsará hacia abajo particularmente a los trabajadores inmigrantes, como única forma de mantenerse, lo que derivará en consecuencia en una particular tensión en las relaciones internas del sector. En este sentido, trabajos clásicos en Psicología Social, como por ejemplo los de Sherif y Sherif (1979), ya prestaron especial atención a los aspectos conflictivos que han surgido en la relación entre grupos y a la reducción de dichos conflictos. Estos estudios están representados por dos teorías fundamentalmente, la primera denominada Teoría de Conflicto Realista de Grupo (TCR), y, la segunda, la Teoría de la Identidad Social (TIS).

La TCR sostenía que el conflicto entre los grupos se originaba al entrar en competición por determinados recursos escasos (Campbell 1965). Investigaciones posteriores demostraron que, efectivamente, las metas de los grupos eran el principal determinante del comportamiento intergrupal (Huici 1999) y que la competición entre los grupos genera actitudes muy negativas entre ellos, potenciando los prejuicios y los comportamientos hostiles hacia los miembros del exogrupo (Sherif 1966).

Partiendo de la TCR, Stephan y Stephan (2000) desarrollan una nueva teoría que plantea que los prejuicios hacia el exogrupo están motivados porque en determinadas circunstancias los grupos se sienten amenazados. Esta Teoría de la Amenaza Integrada (TAl) diferencia cuatro tipos de amenaza (Amenaza Real, Amenaza Simbólica, Ansiedad Intergrupal y Estereotipos Negativos), de las cuales es importante resaltar que no tienen por qué ser reales sino que puede tratarse de percepciones de posibles amenazas. A través del concepto de Amenaza Real o Realista se describe cómo la escasez de recursos como el empleo, las prestaciones sociales, el acceso a la educación o a la sanidad y la percepción de que su distribución no es la adecuada puede enfrentar a los grupos entre si. Numerosas investigaciones realizadas con grupos muy diversos (inmigrantes en Estados Unidos, Israel o España, enfermos de SIDA o terminales de cáncer, etc.) han dado soporte a la TAI (Stephan y Renfro 2002; Stephan, Renfro y Davis 2008) y efectivamente, demostraron que la amenaza podía ser un predictor de prejuicios hacia los diferentes exogrupos muy significativo. Por lo tanto, los estudios clásicos también apuntan a un posible deterioro de las relaciones intergrupales derivado de la nueva situación que plantea la crisis, en este caso, las desavenencias partirían de los escasos puestos de trabajo ofertados en el sector y de la creciente necesidad de los trabajadores de competir por ellos.

Las modificaciones de las condiciones laborales que se han generado a raíz de la crisis, sobre todo la bajada de los salarios podría ser otro de los motivos que podría haber influido en las actitudes de los grupos del sector. Numerosos estudios económicos realizados en Estados Unidos defienden que las actitudes negativas hacia la inmigración parten de los miedos y las competencias que se generan dentro del mercado laboral (Hainmueller y Hiscox 2010). Sin embargo, no todas las investigaciones realizadas en este campo presentan unos resultados unánimes. Mientras algunos trabajos como los realizados por 
Borjas $(2003 ; 2005)$ ponen de manifiesto los efectos negativos que la llegada de población inmigrante genera sobre los salarios y el empleo de los trabajadores nativos en Estados Unidos, otras investigaciones no concluyen de manera tan negativa, pues Card (2001; 2007) muestra que estos efectos realmente no son tan significativos, e incluso Ottaviano y Peri (2008) hablan de ciertos efectos positivos que la inmigración produce a largo plazo sobre la media salarial de los trabajadores autóctonos. En España, los trabajos de Carrasco, Jimeno y Ortega (2006) y Carrasco y Ortega (2005) no encuentran impactos negativos notables atribuibles a la llegada de inmigración al mercado laboral español, aunque estos estudios fueron realizados durante la época de bonanza económica. Con la crisis los salarios sí se han visto afectados, lo que podría incrementar las actitudes negativas hacia los trabajadores inmigrantes, especialmente si se percibe que ellos han sido los primeros en aceptar las rebajas o incluso asumirlas como estrategia de supervivencia.

Por último, uno de los principales efectos de la crisis del sector de la construcción ha sido el incremento tan acelerado que ha sufrido el desempleo dentro del sector. Presumiblemente, este avance del número de parados también tendría sus repercusiones en las relaciones que se mantienen entre los grupos que componen el sector. En los últimos años, se ha abordado el impacto del desempleo sobre las relaciones sociales de las personas desempleadas, aunque sobre todo centrándose en el deterioro de sus relaciones con sus familiares y en los círculos de amistad más cercanos (Buendía 1989, 1990). Numerosos estudios muestran cómo el desempleo incrementa considerablemente el riesgo de que se produzcan inestabilidad y conflictos en el seno de las familias en las que existe algún miembro desempleado y, además, se describe al desempleo como una de las principales causas que incrementa el aislamiento social (Furåker 2010).

El objetivo general de este trabajo persigue examinar si la crisis actual del sector de la construcción además de efectos económicos negativos también ha producido determinadas consecuencias sociales, si éstas tienen un carácter diferencial, según origen u otros factores, en los grupos implicados (inmigrantes frente a autóctonos) y si han repercutido en las relaciones sociales internas de los trabajadores. Los estudios citados indican que la crisis ha supuesto una transformación del contexto de acogida, volviéndolo más hostil, Atendiendo a estos autores, se debe esperar un impacto mayor sobre los trabajadores inmigrantes y un deterioro de las relaciones intergrupales en el seno del sector, en un entorno de mayor competencia, que cada grupo afronta con estrategias que pueden ser diferentes y en el que las actitudes empresariales resultarán decisivas. Igualmente es esperable un aumento de la conflictividad en las relaciones intergrupales y que se manifiesten más intensamente siguiendo líneas de fractura en función del origen étnico, pero también en función de la posición ocupada en el sector (por cualificación, antigüedad o situación laboral de parado o empleado).

Una investigación de esta índole permitirá detectar la existencia de posibles conflictos, los argumentos que los sostienen, y planificar en consecuencia intervenciones más precisas a este respecto.

\section{Metodología}

Para el trabajo de campo se han realizado cuatro grupos de discusión y once entrevistas en profundidad, todas realizadas en la provincia de Almería. Se eligieron dichas técnicas cualitativas con la finalidad de poder recoger y reflexionar sobre las opiniones y percepciones de las personas protagonistas del sector. Los grupos de discusión nos han aproximado a las impresiones e ideas que los trabajadores, tanto españoles como extranjeros, tienen sobre el desarrollo de las relaciones sociolaborales de los grupos de trabajadores entre sí y también de su relación con los empresarios. Las entrevistas han sido realizadas a empresarios del sector considerados informantes claves. Se pretendía complementar los discursos aportados por los trabajadores y mostrar la visión de los empresarios. 


\subsection{Participantes}

La selección de los participantes se ha realizado en función de su relación laboral con el mundo de la construcción y se ha efectuado mediante un muestreo intencional.

De los cuatro grupos de discusión, dos han sido con trabajadores inmigrantes y dos con trabajadores autóctonos. En ambos casos se ha diferenciado entre las categorías laborales de oficiales y peones. Las nacionalidades presentes en los grupos de discusión realizados con inmigrantes han sido: marroquí, ecuatoriana, búlgara, lituana, peruana, hondureña y mauritana. Con el objetivo de obtener la máxima heterogeneidad entre los participantes de los cuatro grupos, se han incluido trabajadores que cuando se realizaron los grupos estaban desempleados y otros en situación de alta laboral.

Las once entrevistas en profundidad se realizaron a empresarios y representantes de los departamentos de Recursos Humanos de empresas representativas del sector de la construcción. Se ha intentado reflejar la diversidad de empresas que configuran el sector, tanto en tamaño (pequeña/mediana: hasta 30 empleados, y grande: desde 31 empleados) como en el tipo de trabajo que realizan (constructoras/promotoras, reformas y obra civil).

\subsection{Variables e instrumentos}

Tanto los grupos de discusión como las entrevistas se han realizado utilizando guiones diseñados ex profeso. Las entrevistas han contado con un único guión para la realización de todas ellas, mientras que para los grupos de discusión se han utilizado dos versiones distintas, uno específico para los grupos de trabajadores autóctonos y otro para los de inmigrantes.

\subsection{Procedimiento}

La selección de los trabajadores para los grupos de discusión en situación activa de empleo se realizó mediante contacto directo visitando numerosas obras y empresas en la provincia de Almería. Los trabajadores en situación de desempleo fueron localizados a través del servicio de orientación sociolaboral Interlabora, gestionado por Cruz Roja Española en Almería.

El contacto con los empresarios se realizó a través de dos vías. Por contacto directo, sin intermediarios, mediante llamadas telefónicas y correos electrónicos. Y, por redes de conocidos, que facilitaban el acercamiento a través de una tercera persona.

Los grupos de discusión se realizaron en la Universidad de Almería. La mayoría de las entrevistas fueron realizadas en las oficinas de las empresas de los entrevistados.

\section{Resultados}

Con la intención de valorar si las transformaciones que ha sufrido el sector en los últimos años han influido en las relaciones mantenidas por sus protagonistas, en primer lugar, nos centraremos en las relaciones mantenidas entre los diferentes grupos en la época anterior a la crisis. Posteriormente, se analiza la situación actual, haciendo especial hincapié en los posibles cambios que hayan sufrido estas relaciones. 


\subsection{Las relaciones intergrupales en la época anterior a la crisis}

\subsubsection{Las relaciones entre los trabajadores}

Una de las características que define el sector de la construcción es la gran diversidad de empresas que lo configuran. Es un sector muy complejo y amplio formado por empresas de diferentes tamaños y de una amplia gama de actividades. Tradicionalmente el sector de la construcción ha admitido también bastante variedad respecto al pago y negociación de los salarios, siendo habitual las retribuciones del trabajo por unidad de obra (destajo). Este tipo de retribuciones facilita la negociación individual de los salarios por parte de los trabajadores y, en ocasiones, permite que se produzcan diferencias en los sueldos de trabajadores que realizan una misma actividad. Esta idiosincrasia ha sido el motivo de que a lo largo de los años en el sector se haya vivido cierta competitividad constante entre los trabajadores. Sin embargo, aunque esto ha marcado a los trabajadores e incluso a las empresas, nunca ha llegado a plantear graves conflictos en las relaciones:

"Esa competencia de decir: 'Oye, que yo te puedo hacer un presupuesto y cobrarte un precio y otro llegar y darte otro precio mas bajo... o más alto, ¿no?'... yo creo que eso siempre ha pasado. Ahora y antes... siempre ha pasado eso" (Empresa 3).

En los años de expansión del sector, las condiciones laborales de los trabajadores mejoraron considerablemente. La abundancia de trabajo relajó el temor a no tener un puesto de trabajo, y dicha competitividad casi desapareció. Las relaciones sociales que se desarrollaron durante este periodo estuvieron marcadas por la tranquilidad y la falta de rivalidad. Las condiciones laborales eran tan buenas, los sueldos tan altos y había tanto trabajo que la competencia entre trabajadores pasó a un segundo plano:

"Cuando la construcción estaba en todo su apogeo, los sueldos de la construcción no eran equiparables a muchos... la mano de obra especializada ganaba, creo que en realidad lo que quería..." (Empresa 9).

Con la llegada de la inmigración las relaciones sociales en el sector sufrieron un cambio, aparecieron nuevos grupos. Los trabajadores inmigrantes, en general, fueron bien aceptados por los trabajadores autóctonos que no vieron peligrar sus puestos de trabajo.

La relación que mantenían los trabajadores entre ellos, cercana o distante, dependía, al parecer, del tamaño de la empresa y de la obra en que estuvieran trabajando. Las empresas pequeñas, con plantillas más reducidas, ofrecían una mayor posibilidad de relación entre las personas de distintas nacionalidades. El pequeño grupo que configuraba la plantilla permitía un contacto más directo y más estrecho entre los trabajadores. En ocasiones, este contacto derivaba en relaciones de amistad. Se trataba de un trato más personal, donde el contacto diario y la colaboración en el trabajo conseguían que se reconociera al individuo como un miembro preferente del otro grupo:

"Tienes que tener en cuenta que si uno trabaja diez horas y media al día, todos los días con esa persona, uno convive más con esa persona que con su propia familia. Porque son cinco días a la semana. Entonces quieras o no, pues bueno, se va tomando un poco de afecto mutuamente, tanto de uno para él como de él para uno" (Oficial Ex5 (1). GOEx (2)).

Sin embargo, conforme el tamaño de la empresa crecía también aumentaba la posibilidad de que se contrataran grupos más grandes de trabajadores de una misma nacionalidad. En ese caso, la separación entre los trabajadores de distintas nacionalidades resultaba evidente e incrementaba la distancia en la relación de los diferentes grupos. En general, las relaciones se limitan estrictamente al plano profesional y rara vez traspasan hacia el plano más personal. Incluso en el plano laboral se marcan grandes distancias, prácticamente sin comunicación. Aún así, las relaciones se describen como tranquilas y sin problemas: 
"Yo estuve aquí en Almería en una obra grande, muy grande, y había collas (3) de ucranianos y había collas de suramericanos y habíamos cuatro o cinco españoles, había más inmigrantes que nosotros. Y hacían eso, se ponían los ucranianos en su corrillo y los suramericanos en otro (GOEs) (4).

Por otro lado, en esta época caracterizada por la serenidad, los únicos trabajadores que dan muestras de que existan algunos conflictos son los trabajadores inmigrantes en sus relaciones con los trabajadores de su misma nacionalidad:

Oficial Ex6: "Para mí, mejor que los marroquíes. Me sabe mal decir eso, porque soy marroquí. Amigos españoles para mí mejor... amigos españoles mejor, compañeros de trabajo, para mí mejor que los marroquíes. Bueno...".

Moderador: ¿Por qué?

Oficial Ex6: "Me están tratando bien, la mayoría, te... por ejemplo, si empieza el trabajo, te, te... explican cómo es el trabajo. Te ayudarán. Y... muchas cosas buenas. Un marroquí no. No te hace eso. Los marroquíes al revés, son muy pocos que son buenos. No te, no te quieren trabajar con ellos".

Oficial Ex5: "Yo..., a mí sí me ha pasado eso, eso sí es verdad. Yo he trabajado, cuando yo empecé a trabajar en la construcción, empecé trabajando con una persona latina, así del mismo país. Y esa persona como que me marginaba".

$(\ldots)$

Oficial Ex5: "Él pensaba que yo le iba a quitar el sitio" (GOEx).

\subsection{Las relaciones entre los trabajadores y los empresarios}

\section{La visión de los empresarios}

Los empresarios mantienen dos tipos de discurso bien diferenciados, en función de dos factores que según ellos determinan el tipo de relaciones que se producen con los trabajadores de sus empresas. Así, existen empresarios para los que la antigüedad del trabajador en la empresa es fundamental, ya que determina la cercanía en el trato entre los trabajadores y el empleador. Y empresarios que consideran que el factor más influyente en la relación de los empresarios con sus trabajadores es la actitud que el trabajador muestra hacia los interesas de la empresa.

Existe un primer discurso en el que los empresarios muestran su preferencia por los trabajadores españoles con los que mantienen una relación que consideran más directa y fluida, consolidada a lo largo de los años que llevan trabajando juntos. A estos se les puede denominar trabajadores "de siempre". La mayor antigüedad en la empresa se usa como justificación de una mejor relación con los autóctonos que con los trabajadores inmigrantes. Argumentan que los vínculos que se han establecido a lo largo de los años generan un trato más próximo, más amigable e incrementa la confianza en el trabajador. En el caso de los inmigrantes, los trabajadores "nuevos", la falta de antigüedad juega un papel bastante negativo que limita las relaciones de los trabajadores con el empresario. La convivencia con los trabajadores inmigrantes se considera más superflua y distante:

"Todos los nacionales, por así decirlo, son, en general, más antiguos que los que hayamos podido incorporar; entonces, claro, las relaciones son distintas, pues no tienes la misma relación, ni conoces lo mismo a una persona con la que llevas trabajando diez años que con otra que llevas ocho meses o diez, o año y medio ¿no? "(Empresa 8).

El segundo discurso representa a empresarios que manifiestan su preferencia por los trabajadores que 
mejor se adaptan a los intereses de las empresas. El perfil de estos trabajadores corresponde a un empleado tranquilo, poco problemático y un tanto flexible respecto a las condiciones laborales que la empresa les ofrece, por lo que se les puede denominar "trabajadores dóciles". Frente a este perfil de trabajadores "dóciles" encontramos otros trabajadores más inflexibles con sus condiciones laborales, que miran más por sus intereses propios que por los de su empresa. A estos trabajadores les hemos denominado "reivindicativos". En este discurso los empresarios muestran su predilección por los trabajadores "dóciles" que, por lo general, son identificados con los extranjeros, y son descritos como menos exigentes y menos conflictivos que los españoles. No todas las nacionalidades tienen la misma consideración, ya que, entre inmigrantes, los que se consideran más difíciles en su trato son los de nacionalidad marroquí. Los trabajadores españoles, sin embargo, se consideran más intransigentes y con posturas menos coherentes. Los empresarios toman como justificación esta actitud hacia el trabajo y hacia la empresa de los trabajadores extranjeros para describir unas relaciones más cordiales y calmadas que las que han podido mantener con algunos trabajadores nacionales, los cuales en ocasiones resultan bastante problemáticos:

"Muy poco problemáticos".

Entrevistadora: ¿Muy poco problemáticos?

"Sí, sí, muy, más dialogantes... hay españoles que... ya no, pero en su época muy... (...) Unos agresivos... algunos... y en cambio los extranjeros... no, nunca" (Empresa 4).

En ambos discursos se pone de manifiesto las buenas relaciones mantenidas por los trabajadores y los empresarios. No se perciben situaciones de conflicto ni enfrentamientos de carácter serio:

"Aquí no hemos tenido ningún tipo de problema en ese sentido, afortunadamente. Ya te digo que es que el clima laboral..., por lo menos, lo que llega aquí, yo no sé si luego entre ellos...cómo se queda la cosa, pero el clima laboral afortunadamente es bastante bueno" (Empresa 5).

\section{La visión de los trabajadores}

La visión que los trabajadores del sector tienen de sus relaciones con los empresarios es más desfavorable que la presentada por los propios empresarios. No se detectan grandes diferencias entre la percepción que muestran los trabajadores españoles respecto a la de los extranjeros, aunque estos últimos son más moderados en sus manifestaciones y se muestran más proclives a mantener una buena relación con sus empleadores. En ambos casos, los trabajadores, y particularmente, los españoles, transmiten una visión del sector en el que se desarrollan unas relaciones mucho más distantes y hostiles, marcadas sobre todo por la indiferencia:

Peón Es2: “¿La relación con nuestros jefes?... venirse a echar una ojeada por la obra que todo mundo esté trabajando y al final de mes si cobras bien y si no, no cobras, pero dentro de lo demás... al final de mes nada más. Esa es la única relación que tiene uno".

$(\ldots)$

Peón Es6: "Mientras cobras va bien, en el momento que no cobras, ya, la relación... malamente" (GPEs) (5).

Oficial Ex5: "Yo todavía conservo amistad con ellos, la verdad que sí. (...) Todavía tengo contacto con él. Que ahora mismo no puede dar trabajo, porque está en la misma situación que todo el mundo".

$(\ldots)$

Oficial Ex7: "Mi jefe; yo me siento con él en la cafetería, te tomas un café con él. Hablamos, entro en su casa, con su familia, igual" (GOEx).

El discurso de los trabajadores muestra la influencia que tiene la duración de la relación contractual del trabajador con la empresa en las relaciones que se establecen con el empleador. Los discursos de los 
trabajadores, tanto autóctonos como inmigrantes, coinciden plenamente con la valoración que se extraía del discurso de los empresarios que preferían relacionarse con los "trabajadores de siempre". Según sus apreciaciones, los trabajadores que han permanecido contratados durante periodos más largos en una misma empresa son los que han mantenido unas relaciones más positivas con los empresarios

El tamaño de las empresas es considerado por los trabajadores como un factor también decisivo que determina la relación que se establece entre trabajadores y empresarios. En las empresas más pequeñas el empresario mantenía una relación más directa con los trabajadores, lo que permitía un contacto más personal, con relaciones más fluidas que derivaban, en ocasiones, en amistad.

\subsection{La aparición de conflictos}

La llegada de la crisis al sector de la construcción ha traído consigo una modificación en las relaciones que se mantienen entre los diferentes grupos que configuran el sector, sobre todo entre los grupos de trabajadores. Este cambio de las relaciones se evidencia claramente en los discursos recogidos y pone de manifiesto numerosas muestras de hostilidad y conflicto de unos grupos hacia otros que hasta entonces no se habían detectado:

"Nos daban lástima y decíamos: 'pues que trabajen, que trabajen'. Claro, cuando ha venido la crisis ahora, ahora es cuando nos hemos dado cuenta de lo que se nos ha metido aquí" (GPEs).

Los cambios más drásticos, tal y como se manifiestan en sus discursos, los sufren los trabajadores españoles. Sus discursos indican un giro hacia unas relaciones más negativas de las que mantenían anteriormente y ponen de manifiesto su percepción de que la nueva situación les obliga a "defender" los pocos puestos de trabajo que van quedando en el sector y la intensificación de la competitividad entre los trabajadores:

"Es que todo el mundo te va a decir lo mismo, hasta que llegaron ellos [trabajadores inmigrantes], nos creíamos todo el mundo que íbamos a trabajar toda la vida" (GPEs).

Se diferencian dos discursos en los trabajadores españoles: el del trabajador español "moderado" y el del trabajador español "frustrado". Ambos discursos mantienen una misma línea de negatividad y muestran el creciente deterioro de las relaciones, pero entre sí presentan dos perfiles de trabajadores diferentes que marcan la intensidad de sus discursos.

\section{Dos perfiles, dos discursos}

Bajo la denominación del español "moderado" está un empleado de la construcción que durante le época de expansión del sector trabajaba en medianas o pequeñas empresas dedicadas, por lo general, a la construcción de pequeñas promociones de viviendas o de viviendas unifamiliares o también podían llevar a cabo rehabilitaciones o reformas. Estas empresas, a pesar de haber sufrido la crisis de manera muy intensa, la han sufrido de un modo más escalonado. Por lo que el trabajador "moderado" actualmente puede ser que esté parado, pero no desde hace mucho tiempo o incluso, puede ser que se mantenga trabajando aunque con unas condiciones laborales muy inferiores a las del periodo anterior. Pese a que sus circunstancias todavía no muestran unas necesidades extremas, su situación económica ha empeorado mucho respecto a los años anteriores en los que las condiciones laborales eran muy buenas. Sus relaciones con los trabajadores inmigrantes durante la época anterior fueron bastante intensas pues tuvo la oportunidad de trabajar con compañeros de diferentes nacionalidades que trabajaban en sus mismas empresas.

El español "frustrado" responde a la descripción de un trabajador que durante el periodo anterior 
pertenecía a empresas grandes dedicadas casi exclusivamente a la construcción de grandes promociones de viviendas. Generalmente se trataba de personal que trabajaba a destajo. Esto les permitió disfrutar de unos sueldos muy elevados y de unos horarios muy flexibles en el periodo anterior a la crisis, con unas condiciones laborales excelentes. Estas grandes empresas fueron las primeras en acusar el frenazo tan brusco del sector y se vieron obligadas de repente a reducir su personal. Por ese motivo muchos de estos trabajadores llevan ya más de dos años desempleados. Su situación actual económicamente es bastante delicada. Algunos han agotado sus prestaciones y han pasado a ser desempleados de larga duración, y, en muchos casos, están sobreviviendo con el subsidio social. En un periodo muy corto su situación económica ha empeorado radicalmente. Según sus manifestaciones, en las empresas en las que trabajaban anteriormente también se contrataban trabajadores inmigrantes pero la relación que mantenían con ellos era bastante distante.

Aunque los dos discursos mantienen una misma tendencia hostil, son los trabajadores españoles "frustrados" los que presentan mayor radicalización en la agresividad de sus discursos. El español "moderado" describe la situación vivida en los últimos años como insostenible y reconoce que la crisis actual, en parte, es una clara consecuencia de los "excesos" llevados a cabo en el sector durante la pasada época (los salarios se dispararon alcanzando cifras impensables, los trabajadores cambiaban de empresas continuamente ofreciéndose a la que le pagara un sueldo más alto, se admitían pagos y cobros en dinero negro, etc.). El español "frustrado" piensa en la época anterior como en una "época dorada" y está obsesionado con volver a esa situación anterior. En contra de lo que a ellos les gustaría, reconocen que aunque el sector se recupere, difícilmente van a vivir de nuevo una época tan gloriosa como la de los años anteriores a la crisis, lo que provoca que su frustración aumente cada vez más. En su discurso culpabilizan de la situación actual del sector: al gobierno, a las empresas, a los bancos y sobre todo, a los trabajadores inmigrantes que no solo han conseguido que el trabajo se terminara antes sino que, actualmente, son una carga que no beneficia la recuperación del sector.

Los puestos ocupados por los trabajadores extranjeros, a pesar de ser menos que los que ocupan los españoles, cada vez se hacen más visibles para estos últimos. El discurso de los españoles manifiesta abiertamente su malestar porque los extranjeros "les están robando" un trabajo que ellos merecen más, simplemente por ser españoles. Se les acusa de aceptar sueldos bajísimos y condiciones muy precarias. El hecho de que haya españoles trabajando con malas condiciones laborales no resulta tan molesto para los trabajadores españoles desempleados como el hecho de que sean trabajadores extranjeros los que están ocupando los escasos puestos de trabajo que actualmente ofrece el sector:

"Cuando pase esta crisis, que pasará, el ochenta por ciento de, de los trabajadores que queden en la construcción, porque vamos a quedar muy pocos, los que queden van a ser inmigrantes. Por una simple razón, porque ellos trabajan más barato, no piden tantas pagas, trabajan más horas extras y aguantan mucho más que nosotros" (GOEs).

La llegada de la crisis no solo ha supuesto grandes pérdidas económicas a los trabajadores de la construcción, sino que también les ha supuesto un retroceso en su estatus laboral. Hoy en día, se ven obligados a aceptar todo tipo de trabajos dentro del sector. En algunos casos ha significado que se han visto obligados a realizar trabajos para los que estaban sobrecualificados; en otros casos, han tenido que aceptar puestos de categoría inferior a la suya, o, lo que es peor, han tenido que realizar su trabajo pero aceptando contratos de categoría inferior. Ante esta pérdida de estatus laboral, los trabajadores "moderados" y los "frustrados" presentan dos reacciones diferentes. Por un lado, los "moderados" ven estos hechos como una necesidad de adaptarse a las nuevas circunstancias del sector, se muestran más conformistas, aunque critican duramente a las empresas, pues consideran que están aprovechando la nueva coyuntura para rebajar costes a costa de los sueldos de los trabajadores. Por su parte, los "frustrados" culpabilizan a los trabajadores inmigrantes. Consideran que los trabajadores inmigrantes aceptan cada vez peores condiciones laborales y esto anima a las empresas a empeorar las condiciones de todos los trabajadores del sector: 
"A mí no me va a llegar ese tío a pagarme sesenta euros cuando tiene a rumanos... que por treinta euros están trabajando" (GOEs).

Los discursos de los trabajadores extranjeros también han ido enrareciéndose y, actualmente, denotan cierta crispación. En este caso no es debido a que ellos se sientan amenazados por los trabajadores españoles, ya que la competencia de los trabajadores extranjeros suele producirse entre los trabajadores de su misma nacionalidad o entre extranjeros de otras nacionalidades, sino por el malestar que les provoca el ser conscientes de que los trabajadores españoles les consideran una amenaza, ante lo que muestran una creciente preocupación:

Peón Ex7: "Dicen que le estamos quitando un puesto de trabajo a los españoles".

Peón Ex8: "Eso pueden decir ahora, pero más antes, el trabajo, lo hacíamos los extranjeros, lo que ellos rechazaban lo hacíamos nosotros" (GPEx) (6).

\section{Conclusiones}

\subsection{Situación previa a la crisis: periodo de calma y tranquilidad}

Durante la época expansiva del sector de la construcción, las relaciones intergrupales que se mantuvieron entre los trabajadores de las diferentes nacionalidades parecen haber sido cordiales, aunque un tanto distantes. Se observa una clara tendencia a agruparse por nacionalidades, aunque los años de convivencia en el trabajo pudieran dar lugar a cierta cercanía entre los trabajadores de diferentes procedencias.

Sin embargo, sí se detecta cierta tensión entre los trabajadores inmigrantes. El inmigrante que había conseguido establecerse dentro del sector de la construcción presentaba una actitud más negativa hacia sus propios compatriotas que hacia los trabajadores españoles. La teoría nos facilita dos justificaciones para esta actitud:

- Consideraban que sus compatriotas tenían una formación y unas características tan similares a las suyas que podían desplazarles de sus trabajos más fácilmente que los trabajadores españoles. Les consideraban competencia directa. Tal y como expone la TCR (Sherif 1966) entre los grupos de estatus similar se incrementa la competencia, y más en los casos en los que el grupo presenta un marcado carácter hermético, es decir, cuando cambiar de grupo social resulta muy complicado, como en el caso que nos ocupa.

- Los grupos más desfavorecidos generalmente no entran en conflicto con los grupos dominantes, sino con los de su mismo estatus. Contrariamente a lo que se podría pensar, estos grupos consideran las desigualdades en el reparto de los recursos como algo normal y legítimo y suelen mostrarse bastante negativos hacia su propio grupo (Apfelbaum 1979). Esta podría ser también otra causa por la que los trabajadores inmigrantes se muestran más negativos hacia su propio grupo ya que, hasta cierto punto, considerarían normal que los trabajadores autóctonos obtuvieran, quizás, un puesto de trabajo mejor que el de ellos o que sus condiciones laborales estuvieran mejoradas. Sin embargo, esto no lo tolerarían respecto a los trabajadores inmigrantes, pues los consideran dentro del grupo desfavorecido igual que ellos.

Al analizar las relaciones que mantenían los trabajadores y los empresarios también podrían describirse en la misma línea de serenidad. No se detecta ningún síntoma de conflicto importante, aunque de manera sutil se entrevé la preferencia que muestran los empresarios por relacionarse con un grupo de trabajadores o con el otro. Analizando sus propias manifestaciones, entre los empresarios podemos diferenciar dos discursos muy marcados en función de los factores que determinan estas preferencias: 
- Por un lado, los que consideran la antigüedad de los trabajadores en la empresa como el factor determinante de las buenas relaciones. En este caso, los empresarios que mantienen este primer discurso, defienden un trato más cercano con los trabajadores "de siempre", refiriéndose de esta manera a los españoles que han acumulado más años a su servicio. Esta antigüedad ha favorecido el nacimiento de vínculos afectivos más estrechos.

- Por otro lado, los que justifican las buenas relaciones en función del grado de implicación que el trabajador muestra con los intereses de la empresa y la actitud que tiene hacia su propio trabajo. Estos empresarios muestran claramente su preferencia por los trabajadores que ellos consideran "más dóciles", que muestran mayor implicación y que no desarrollan conductas de exigencia laboral, resultando en este caso actitudes más favorables hacia los trabajadores extranjeros. Aquí los trabajadores inmigrantes están en clara desventaja respecto a los nativos, puesto que, en comparación con estos, los extranjeros precisamente destacan no solo por tener menor antigüedad, sino también, por que un amplio porcentaje de ellos trabajan a media jornada o tienen contratos temporales (Carrasco y Ortega 2005), lo que les permite menos margen de relación con el empresario.

En ambos discursos los estereotipos juegan un papel fundamental en las relaciones sociales mantenidas y aparecen como justificación de las relaciones positivas o negativas. La carga de generalizaciones es muy elevada en estos discursos. No solo hacen referencia a las actitudes y comportamientos de los trabajadores autóctonos e inmigrantes ante la empresa, sino que dentro del grupo de los inmigrantes establecen marcadas diferenciaciones estereotipadas entre las distintas nacionalidades. Así, por ejemplo, los trabajadores latinos son vistos como los más educados, por lo que es muy sencillo mantener un trato afable con ellos, mientras que los marroquíes son considerados más ariscos en su comunicación con los empresarios.

Por último, al reflexionar sobre los discursos de los trabajadores, respecto a la proximidad que mantenían con sus empleadores ambos grupos, tampoco se aprecian síntomas de conflicto. Sin embargo, sí se perciben unas relaciones mucho más displicentes y distantes que las que se deducen de los discursos de los empresarios. Para los trabajadores la antigüedad en la empresa es un factor fundamental y muy favorable. Igualmente destacan como en las empresas pequeñas se crean unos vínculos más cercanos entre el empresario y el trabajador al margen de sus nacionalidades.

\subsection{La crisis está modificando las relaciones interpersonales}

La situación de crisis está provocando una dura competencia entre los trabajadores que se ven obligados a luchar por los limitados recursos que actualmente oferta el sector. Algunos de los estudios (Rinken, Escobar y Velasco 2011; Cea y Valles 2009; 2010; Fix y otros 2009 y González 2008), ya adelantaban la competencia como uno de los resultados sociales de la crisis. El principal factor que exacerba esta competencia, y, consecuentemente, el cambio en las relaciones laborales de los distintos grupos, es el desempleo. Ninguno de los temores antes descritos tiene comparación con el miedo que sienten los trabajadores a quedarse sin empleo y todo lo que ello implica.

En este escenario, la nacionalidad de los trabajadores está adquiriendo una notable relevancia en el desarrollo de las relaciones intergrupales en el trabajo. El aumento de la competencia por los escasos empleos, unido a la creciente percepción que tienen los trabajadores españoles de que los extranjeros trabajan aceptando peores sueldos y condiciones laborales que ellos, acrecienta la sensación de amenaza laboral, llamada amenaza a nivel realista (Ybarra y Stephan 1994). Tanto el estudio realizado por Ybarra y Stephan (1994), como los demás estudios sociales dedicados a valorar el impacto de la inmigración económica definen la amenaza como un potente predictor de reacciones negativas. Actualmente la sensación de amenaza percibida por los trabajadores españoles está originando sentimientos y reacciones negativas hacia los trabajadores inmigrantes, quienes son percibidos por los autóctonos como un lastre para el sector. 


\subsection{Las nuevas actitudes}

Una de las principales aportaciones de esta investigación reside en que se han detectado dos discursos diferentes articulados por los trabajadores españoles ("español frustrado" y "español moderado"). Cada uno de estos discursos está adscrito a un perfil determinado de trabajador que tiene unas características concretas respecto al tipo de empresa en la que trabajó durante el periodo de bonanza, cómo eran sus relaciones con sus compañeros extranjeros y si están o no desempleados actualmente.

En el primer grupo, los trabajadores están obsesionados con la época de esplendor que ha vivido el sector en los últimos años aunque reconocen que es improbable que se vuelva a producir. Sus circunstancias laborales y económicas, actualmente, son muy malas en la mayoría de los casos. El sector se muestra carente de oportunidades para estos trabajadores. Esto les produce una frustración que aumenta conforme su situación laboral va empeorando. El trabajador "frustrado" se vuelve en contra de los trabajadores inmigrantes a los que culpabiliza de la situación que está atravesando el sector y lo exteriorizan en sus discursos a través de las constantes manifestaciones en contra de la inmigración. Aunque estos trabajadores también manifiestan su malestar con el gobierno, la economía mundial, los bancos, las empresas, consideran que todos son demasiados poderosos y que no van a resolver sus problemas, esto aumenta su frustración. Intentan simplificar el problema y buscar responsables para encontrar una explicación a la difícil situación que están viviendo y culpabilizan a los trabajadores inmigrantes de esta situación, responsabilizándoles incluso del agotamiento del trabajo en el sector de la construcción.

El discurso del "español moderado", a pesar de poner de manifiesto también el cambio negativo que se ha producido en las relaciones y la creciente rivalidad que se vive en el sector, muestra un comportamiento más calmado. Este grupo de trabajadores está sufriendo los efectos de la crisis de manera más escalonada. Muchos de ellos todavía trabajan o no llevan parados mucho tiempo por lo que no están tan acuciados aunque sus condiciones hayan empeorado considerablemente. El español "moderado" en su discurso busca diferentes justificaciones para la nueva situación y no se limita simplemente a culpabilizar a los trabajadores inmigrantes. En general, su discurso es más crítico con la manera de actuar de todo el sector durante el periodo anterior e incluso se lamenta de los excesos que se cometieron.

Por lo tanto, a partir de los discursos de empresarios y de trabajadores podríamos describir cómo se producen actualmente las conexiones entre los distintos grupos que configuran el sector. La figura 1 muestra gráficamente este panorama, que se articula en torno a dos ejes: uno de antigüedad laboral y otro de docilidad/reivindicación. Por un lado, de acuerdo con sus discursos, las mejores relaciones intergrupales son las que mantienen los trabajadores españoles "moderados" y los extranjeros que provienen de Sudamérica, de los países del Este y los subsaharianos, tanto con los empresarios, como entre ellos. Estos grupos de trabajadores, situados en los cuadrantes superiores, a pesar de que hayan surgido algunos conflictos, son los que tienen posturas menos hostiles, menos extremistas y muestran unas actitudes más serenas.

El aumento de la tensión se detecta entre los colectivos de trabajadores "frustrados" y de trabajadores inmigrantes, particularmente los de nacionalidad marroquí (cuadrantes inferiores). Los discursos muestran cómo en las relaciones intergrupales se mantiene una creciente crispación como resultado de un mayor distanciamiento y un ambiente laboral más tenso, lo que podría desembocar en problemas sociales más profundos. 


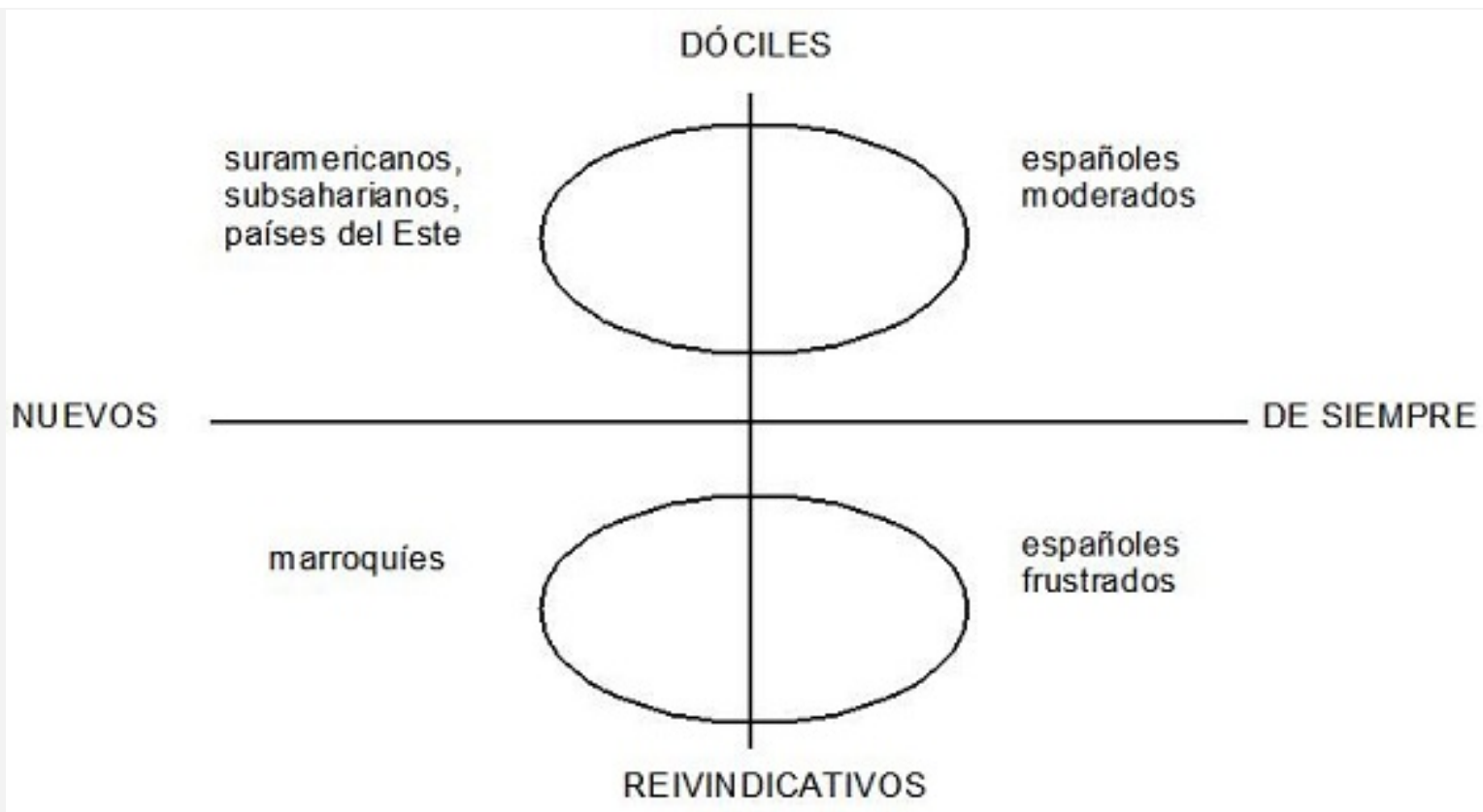

Figura 1. Relaciones intergrupales en el sector de la construcción.

Tanto para los trabajadores españoles "moderados" como para los "frustrados", el hecho de que existan trabajadores extranjeros que actualmente estén trabajando, les resulta muy molesto. A pesar de ser numéricamente muy inferior al de los españoles que se han mantenido trabajando en el sector, cada vez parecen hacerse más visibles. En esto apoyan sus discursos cuando defienden que los inmigrantes les están "robando" unos puestos de trabajo que deberían de estar ocupados por los españoles. Demostrando su favoritismo endogrupal, los trabajadores autóctonos no parecen reparar en el hecho de que seguramente los trabajadores españoles que actualmente tienen un empleo han tenido que rebajar sus sueldos y empeorar sus condiciones laborales, al igual que lo han tenido que hacer los extranjeros. Esta actitud que se les recrimina constantemente a los trabajadores extranjeros parece estar perfectamente admitida en los españoles. Ellos consideran que los españoles tienen que tener cierta prioridad para obtener y mantener los trabajos por el mero hecho de ser españoles.

Por su parte, los trabajadores extranjeros muestran gran malestar porque no son ajenos a los discursos de los españoles. Ellos son conscientes de que se les culpabiliza en parte de la situación que sufre el sector y muestran cierta preocupación hacia posibles situaciones de rechazo. Sin embargo, ellos repiten esas mismas actitudes respecto a los trabajadores de otras nacionalidades.

En conclusión, el escenario actual que presenta el sector de la construcción se caracteriza por la falta de oportunidades que ofrece actualmente para los miles de trabajadores que habían formado parte de él. Las repercusiones que ha tenido la crisis sobre los trabajadores, tanto inmigrantes como autóctonos, están siendo devastadoras. La rápida evolución del sector hacia parámetros tan negativos ha colocado a los trabajadores en una situación económica muy difícil, que está produciendo, a su vez, un deterioro de las relaciones intergrupales. El presente trabajo ha detectado preocupantes manifestaciones de hostilidad con una gran tendencia hacia el empeoramiento si las prestaciones sociales se agotan y las perspectivas de empleo no mejoran en el futuro.

\section{Bibliografía}


Apfelbaum, Erika

1979 "Relations of domination and movements for Liberation: An analysis of power between groups", en William G. Austin y Stephen Worchel (eds.), The Social Psychology of Intergroup Relation. Monterey, Brooks/Cole: 188-203.

Borjas, George J.

2003 "The Labor Demand Curve Is Downward Sloping: Reexamining the Impact of Immigration on the Labor Market", Quarterly Journal of Economics, 118 (4): 1335-1374.

2005 Native internal migration and the labor market impact of immigration. Cambridge, National Bureau of Economic Research.

Buendía Vidal, José

1989 "Aspectos psicológicos y psicopatológicos del desempleo: depresión y apoyo social”, Psiquis, 2: 47-53.

1990 “Psicopatología del desempleo”, Anales de Psicología, 6: 21-36.

Card, David

2001 "Immigrant Inflows, Native Outflows, and the Local Labor Market Impacts of Higher Immigration", Journal of Labor Economics, 19 (1): 22-64.

2007 "How Immigration Affects US Cities", CReAM Discussion Paper, London, Centre for Research and Analysis of Migration, University College London.

Carrasco Perea, Raquel (y Carolina Ortega Masagué)

2005 La inmigración en España: características y efectos sobre la situación laboral de los trabajadores nativos. Madrid, Fundación Alternativas.

Carrasco Perea, Raquel (Juan Francisco Jimeno Serrano y Carolina Ortega Masagué)

2006 El efecto de la inmigración en las oportunidades de empleo de los trabajadores nacionales. Bilbao, Fundación BBVA.

Cea D’Ancona, María Ángeles (y Miguel S. Valles Martínez)

2009 Evolución del racismo y la xenofobia en España. Madrid, Ministerio de Trabajo e Inmigración, Subdirección General de Información Administrativa y Publicaciones.

2010 Living together: Ciudadanía europea contra el racismo y la xenofobia. Madrid, Ministerio de Trabajo e Inmigración, Secretaría de Estado de Inmigración y Emigración, Dirección General de Integración de los Inmigrantes.

Clemente López, Jesús (e Inmaculada García Mainar)

2009 "La crisis y los indicadores del mercado de trabajo", Cuadernos del Mercado de Trabajo, 2: 23-33.

Fix, Michael (y otros)

2009 Migration and Social Recession. Washington, Migration Policy Institute.

Furåker, Bengt

2010 Financial, economic and social crisis: On the social consequences of unemployment. Brussels, Directorate General for Internal Policies, Policy Department A, Economic and Scientific Policies, European Parliament.

González Enríquez, Carmen

2008 Los sindicatos ante la inmigración. Madrid, Ministerio de Trabajo e Inmigración, Subdirección General de Información.

González-Martín, Beatriz (Pablo Pumares Fernández y Antonio J. Rojas Tejada)

2010 El "colapso" del sector de la construcción y sus repercusiones sociolaborales sobre la población 
inmigrante. Análisis de las fuentes estadísticas. Sevilla, Fundación Pública Andaluza Centro de Estudios Andaluces, Consejería de la Presidencia, Junta de Andalucía.

Hainmueller, Jens (y Michael J. Hiscox)

2010 "Attitudes toward Highly Skilled and Low-skilled Immigration: Evidence from a Survey Expetiment", American Political Science Review, 104: 61-84.

http://journals.cambridge.org/action/displayAbstract?fromPage=online\&aid $=7449424$

Huici Casal, Carmen

1999 "Estereotipos", en J. Francisco Morales Domínguez (coord.), Psicología Social. Madrid, McGrawHill: 87-98

Ottaviano, Gianmarco (y Giovanni Peri)

2008 "Immigration and National Wages: Clarifying the Theory and the Empirics", NBER Working Paper 14188. Cambridge, National Bureau of Economic Research

Portes, Alejandro (y Jozsef Böröcz)

1992 "Inmigración contemporánea. Perspectivas teóricas sobre sus determinantes y modos de accesos", Alfoz Madrid, Territorio, economía y sociedad, 91-92: 20-34.

Rinken, Sebastian, (María Soledad Escobar Villegas y Saúl Velasco Dujo)

2011 Opiniones y actitudes de la población andaluza ante la inmigración (III). Más allá del discurso funcionalista. Sevilla, Junta de Andalucía, Consejería de Empleo, Dirección General de Coordinación de Políticas Migratorias.

Sherif, Muzafer

1966 Group conflict and cooperation: their social psychology. London, Routledge and Regan Paul.

Sherif, Muzafer (y Carolyn W. Sherif)

1979 "Research on intergroup relations", en William S. Austin y Stephen Worchel (eds.), The social psychology of intergroup relations. Monterey, Brooks/Cole: 7-18.

Stephan, Walter G. (y Cookie White Stephan)

2000 "An integrated threat theory of prejudice", en Stuart Oskamp (ed.), Reducing prejudice and discrimination. Mahwah, Lawrence Erlbaum: 23-45.

Stephan, Walter G. (y Lausanne Renfro)

2002 "The Role of Threat in Intergroup Relations", en Diane M. Mackie y Eliot R. Smith (eds.), From Prejudice to Intergroup Emotions. Differentiated Reactions to Social Groups. New York, Psychology Press: 191-207.

Stephan, Walter G. (Lausanne Renfro y Mark D. Davis)

2008 "The Role of Threat in Intergroup Relations" en Ulrich Wagner, Linda R. Tropp, Gillian Finchilescu

y Colin Tredoux (eds.), Improving Intergroup Relations. Oxford, Blackwell: 55-72.

Ybarra, Oscar J. (y Walter G. Stephan)

1994 "Amenaza percibida como predictor de prejuicios y estereotipos. Reacciones de los americanos a los inmigrantes mejicanos", Boletín de Psicología, 42: 39-54.

\section{Notas}


Este trabajo ha sido posible gracias al proyecto de investigación La crisis en el sector de la construcción. Repercusiones sociolaborales sobre los trabajadores inmigrantes y los trabajadores autóctonos (PRY 135/09), financiado por la Fundación Pública Andaluza, Centro de Estudios Andaluces de la Consejería de la Presidencia de la Junta de Andalucía.

Parte de este artículo fue presentada en el I Congreso Internacional sobre Migraciones en Andalucía (febrero 2011).

1. Para mantener el anonimato de los participantes de los grupos de discusión se les nombra como "Oficial Es" y "Oficial Ex" para referirnos a los participantes de los grupos de oficiales. Igualmente "Peón Es" y "Peón Ex" harán referencia a los de los grupos de peones. "Es" y "Ex" diferencia entre españoles y extranjeros. El número que aparece es el distintivo de cada uno de los participantes.

2. GOEx: Grupo de discusión de Oficiales Extranjeros.

3. Empresas muy pequeñas que estaban compuestas por un oficial o dos a lo sumo, y sus correspondientes peones.

4. GOEs: Grupo de discusión de Oficiales Españoles.

5. GPEs: Grupo de discusión de Peones Españoles.

6. GPEx: Grupo de discusión de Peones Extranjeros. 